\title{
Nasal tip plasty using three-dimensional printed polycaprolactone (Smart Ball ${ }^{\circledR}$ )
}

\author{
Joo Hyoung Kim ${ }^{1}$, Geon Woo Kim ${ }^{1}$, Won Kyung Kang ${ }^{2}$ \\ ${ }^{1}$ Department of Plastic and Reconstructive Surgery, Pusan National University School of Medicine, Busan, Korea \\ ${ }^{2}$ Bora Plastic and Reconstructive Surgery Clinic, Ansan, Korea
}

Received: June 18, 2019

Revised: August 19, 2019

Accepted: August 22, 2019

Corresponding author:

Won Kyung Kang

Bora Plastic and Reconstructive

Surgery Clinic, 7 Gojan 1-gil,

Danwon-gu, Ansan 15360, Korea

Tel: $+82-31-483-3366$

Fax: +82-51-243-9405

E-mail: gmgunu1101@gmail.com
Background: Rhinoplasty is one of the most commonly performed cosmetic surgery procedures. Most Asians desire elevation of their relatively flat nasal dorsum and tip to make them appear more prominent. This study introduces a simple method of nasal tip plasty using three-dimensional (3D)-printed polycaprolactone (PCL) (Smart Ball $\left.{ }^{\circledR}\right)$, which provides the required length and volume for this purpose and enables the creation of a nasal tip of the desired shape in a safe and simple manner.

Methods: Between September 2014 and May 2017, 22 patients participated in a survey to assess postoperative satisfaction levels. Additionally, three plastic surgeons compared patients' pre- and 1 -year postoperative photographs to evaluate the results. All patients underwent 2- to 4-year postoperative follow-up.

Results: Levels of subjective satisfaction among patients were $3.59,3.50,3.82,3.73,3.55$, and 3.82 for each of the 6 categories evaluated, with a mean of 3.67/4 points, indicating high satisfaction levels. The mean plastic surgeon-reported score for the 22 patients was $4.47 / 5$ points, which also indicates highly successful outcomes. Postoperative nasal tip rotation and tip projection were ideal in most patients.

Conclusion: Our novel method using 3D-printed PCL (Smart Ball ${ }^{\circledR}$ ) provides the optimal length and volume required for nasal tip plasty and enables the creation of a nasal tip of the desired shape, in a safe and simple manner. An advantage of our method is that it retains the original nasal structure in contrast to structural changes observed with the use of conventional methods.

Keywords: Bioprinting; Plastic surgery; Polycaprolactone; Rhinoplasty

\section{Introduction}

Rhinoplasty is one of the most common plastic surgery procedures performed globally; however, the methods that are applicable to or successful in White patients may be unsatisfactory among Asians. This is on account of the differences between Asians and Caucasians with regard to their expectations of surgical outcomes and the distinctive nasal structures of these races, which require different approaches to rhinoplasty [1].
Modern nasal plastic surgery methods used in Asians have evolved with the help of advanced concepts and techniques introduced in the West, owing to greater academic exchanges between the East and the West and better access to articles published in international journals (Fig. 1).

Correction of a deviated nose and unaesthetic nasal shapes requires the application of methods developed by Western medicine; however, performing elaborate surgical procedures is challenging secondary to the anatomical differences between Asian and Cau- 

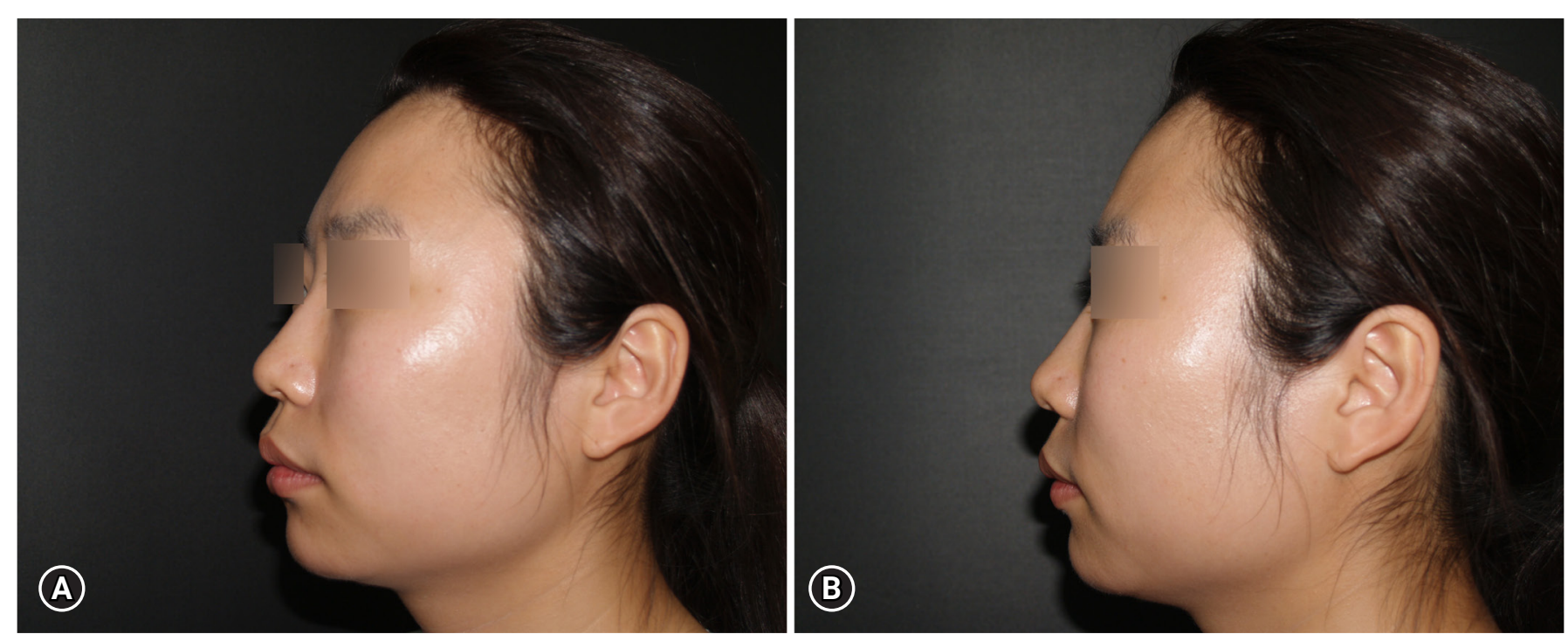

Fig. 1. Photographs show a typical Asian nose with a flat nasal dorsum and tip (Asians typically have thicker skin and a lower nose). (A) Three-quarter view. (B) Lateral view.

casian noses.

Polycaprolactone (PCL) is a synthetic biodegradable aliphatic polyester that undergoes hydrolytic degradation in humans. The end products of PCL degradation do not include harmful elements, such as boron or chlorine. Therefore, PCL is approved by the United States Food and Drug Administration for use in humans owing to its high biocompatibility and safety [2].

Rhinoplasty is usually performed in patients with a deviated nasal septum, a wide lobule with columellar retraction, and deficient nasal tip projection (NTP). In this study, we discuss the successful outcomes of a novel rhinoplasty technique using three-dimensional (3D)-printed PCL (Smart Ball ${ }^{\circledR}$ ), which increases the length and volume of the nasal tip and enables the creation of a nasal tip of the desired shape, in a safe and simple manner.

\section{Materials and methods}

\section{Ethics statement}

This study was approved by the Institutional Review Board of Pusan National University Hospital (IRB No. K-2019-32819369). All patients provided written informed consent for the use and publication of their photographs.

\section{Patients}

This study included 22 patients who underwent nasal tip plasty with 3D-printed PCL between September 2014 and May 2017. Patients who had previously undergone invasive nasal plastic surgery were excluded.

Postoperative results were evaluated based on the following data: (1) a postoperative patient satisfaction survey, (2) objective evaluation of plastic surgeon-reported scores obtained 1 year postoperatively, and (3) measurement of the degree of nasal tip rotation and tip projection.

1) Patient survey as a measure of subjective levels of satisfaction The Rhinoplasty Outcome Evaluation questionnaire (Fig. 2) was distributed to all patients included in the study [3]. Patients responded to 6 questions, and each question was answered on a scale from 0 (lowest satisfaction) to 4 (high satisfaction).

\section{2) Evaluation by plastic surgeons}

Surgical outcomes were evaluated by 3 board-certified plastic surgeons who compared patients' pre- and 1-year postoperative photographs. Outcomes were expressed as a score rated on a scale of 1 to 5 as follows: highly successful (5 points), moderately successful (4 points), successful (3 points), somewhat successful ( 2 points), and unsuccessful ( 1 point).

3) Measurement of the degree of nasal tip rotation and tip projection

Pre- and postoperative photographs obtained in 22 patients were analyzed with regard to the nasolabial angle, columellar lobular angle, and NTP. The nasolabial angle is the angle between a line drawn through the anterior and posterior ends of the nostril and the vertical facial plane (Fig. 3A) [4]. The columellar-lobular angle is formed at the junction of the columella and the infratip lobule and represents the junction of the middle and medial crura (Fig. 3B). Tip projection is defined as the distance from the alar 


\section{Rhinoplasty outcomes evaluation (ROE)}

This questionnaire is designed to assist your surgeon in determining the best patient outcomes following rhinoplasty surgery. Your comments are confidential and may be used to refine surgical procedures for future patients. Please circle the number that best characterizes your current opinion regarding the following questions:

1. How well do you like the appearance of your nose?

$\begin{array}{ccccc}\text { Not at all } & \text { Soemwhat } & \text { Moderately } & \text { Very much } & \text { Completely } \\ 0 & 1 & 2 & 3 & 4\end{array}$

2. How well are you able to breathe through your nose?

$\begin{array}{ccccc}\text { Not at all } & \text { Soemwhat } & \text { Moderately } & \text { Very much } & \text { Completely } \\ 0 & 1 & 2 & 3 & 4\end{array}$

3. How much do you feel your friends and loved ones like your nose?

$\begin{array}{ccccc}\text { Not at all } & \text { Soemwhat } & \text { Moderately } & \text { Very much } & \text { Completely } \\ 0 & 1 & 2 & 3 & 4\end{array}$

4. Do you think your current nasal appearance limits you social or professional activities?

$\begin{array}{ccccc}\text { Always } & \text { Usually } & \text { Sometimes } & \text { Rarely } & \text { Never } \\ 0 & 1 & 2 & 3 & 4\end{array}$

5. How confident are you that your nasal appearance is the best that it can be?

$\begin{array}{ccccc}\text { Not at all } & \text { Soemwhat } & \text { Moderately } & \text { Very much } & \text { Completely } \\ 0 & 1 & 2 & 3 & 4\end{array}$

6. Would you like to surgically alter the appearance or function of your nose?

$\begin{array}{ccccc}\text { Definitely } & \text { Most likely } & \text { Possibly } & \text { Probably not } & \text { No } \\ 0 & 1 & 2 & 3 & 4\end{array}$

Fig. 2. The Rhinoplasty Outcomes Evaluation survey administered to patients.
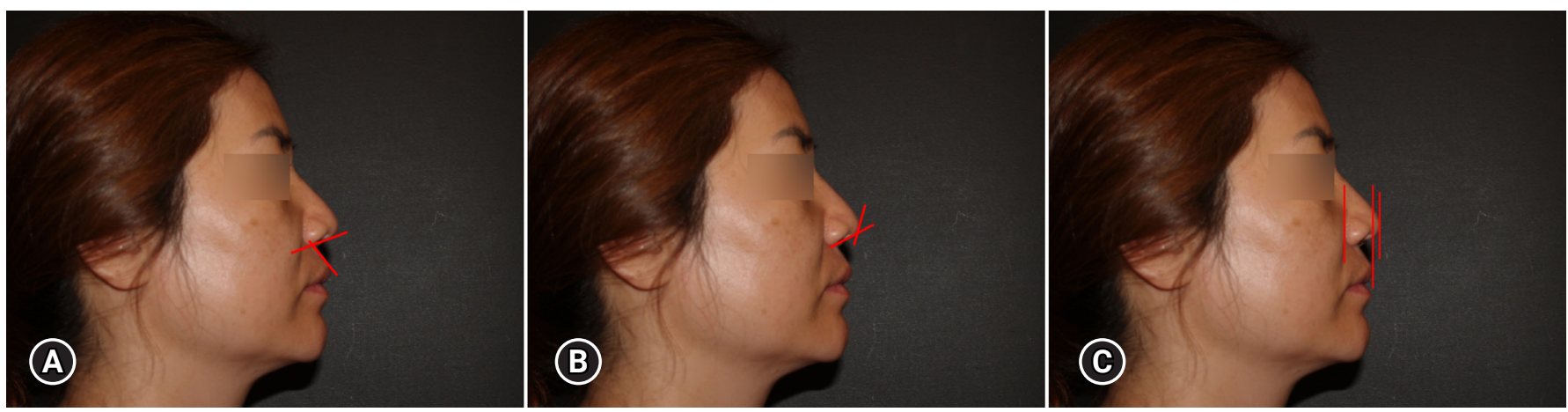

Fig. 3. Measurement of the degree of nasal tip rotation and tip projection. (A) Nasolabial angle. (B) Columellar-lobular angle. (C) Tip projection. 
base to the nasal tip (Fig. 3C).

\section{Surgical procedure}

Surgical draping of the facial area was performed using the standard method followed in clinical practice. Subsequently, we marked the area where we planned to insert the 3D-printed PCL (Smart Ball ${ }^{\circledR}$ ) (Fig. 4). It is important to remember that in addition to achieving a sharp nasal tip, the tip should be in the correct position, because following this procedure, the nose may appear slightly longer or shorter.

All procedures were performed under local anesthesia. After the creation of an infra-cartilaginous incision (Fig. 5), curved or straight Metzenbaum scissors were used for dissection. If dissection is performed too close to the skin, the implant or the thick cartilage remains in continuous contact with the thin skin, thereby affecting the blood circulation and causing skin discoloration and/or thinning, which may complicate the surgery. Therefore, careful dissection is important to avoid close contact between the implant and the skin.

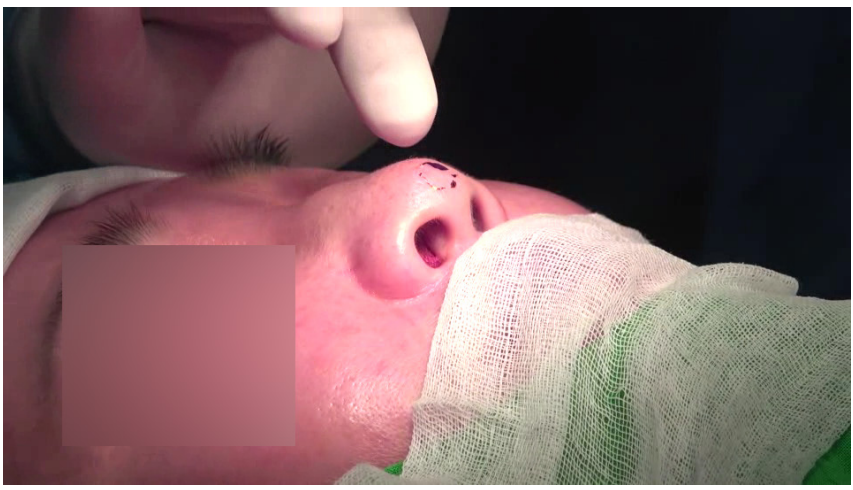

Fig. 4. Preoperative markings in the area of implant insertion. Three-quarter view.
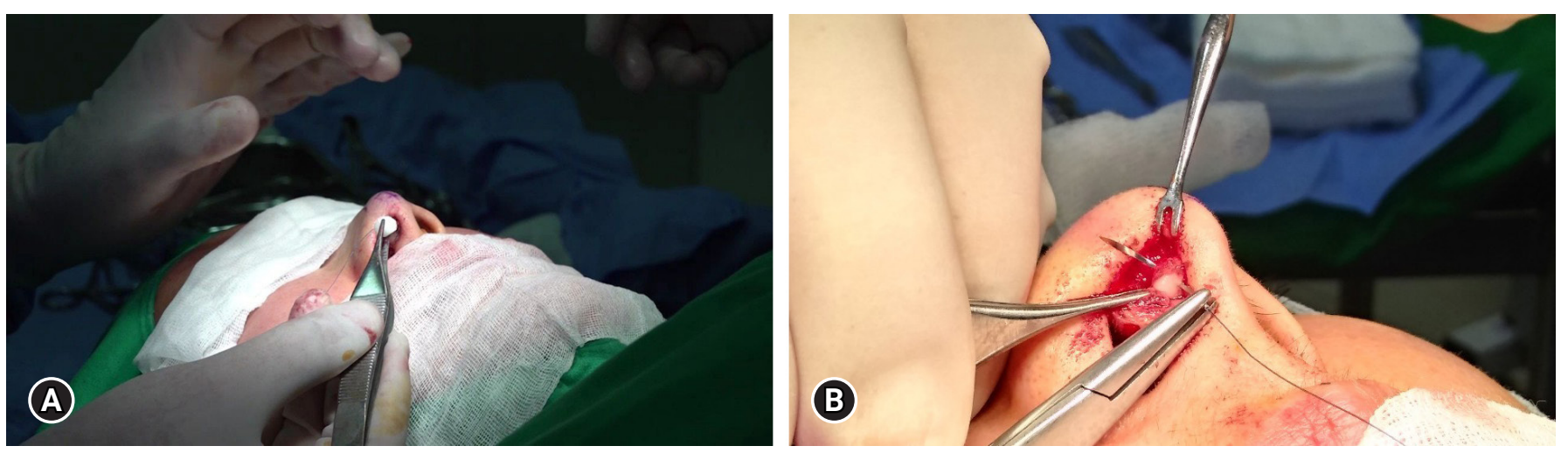

Fig. 6. (A) Measurement of the size of Smart Ball ${ }^{\circledR}$ after trimming it to the appropriate size. (B) Fixation of the Smart Ball® implant using a suture and cutting the remaining suture before insertion. 
The incision may be sutured with the same material that is used for conventional augmentation rhinoplasty, and absorbable or nonabsorbable sutures are acceptable. This technique is associated with minimal postoperative edema; therefore, application of a light dressing over 3 days postoperatively is sufficient.

\section{Results}

Of the 22 patients included in the study, 6 were men and 16 were women. All patients underwent 2- to 4-year postoperative follow-up (mean 36 months).

\section{Patient survey as a measure of subjective levels of satisfaction} Surveys performed in the 22 patients who underwent this procedure indicated excellent postoperative satisfaction, with a mean score of 3.67 (Table 1).

\section{Evaluation by plastic surgeons}

Comparative evaluation of patients' pre- and postoperative photographs performed by 3 plastic surgeons indicated successful outcomes in all 22 patients included in the study, with a mean score of 4.48 (Table 2).

\section{Measurement of the degree of nasal tip rotation and tip projection}

Pre- and postoperative photographs obtained in 22 patients were analyzed with regard to measurements of the nasolabial angle, col-

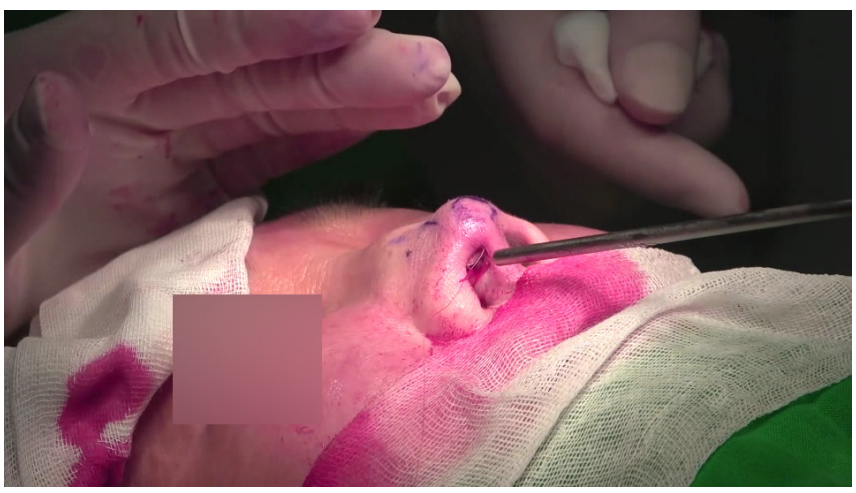

Fig. 7. Photographs obtained immediately after insertion of the Smart Ball ${ }^{\circledR}$ implant. Three-quarter view. umellar lobular angle, and tip projection (Table 3).

Notably, $95^{\circ}-100^{\circ}$ and $45^{\circ}$ are considered ideal measurements for the nasolabial and columellar lobular angles, respectively. Tip projection is considered normal if 50\%-60\% of the nasal tip lies anterior to the vertical line adjacent to the most prominently projecting portion of the upper lip. Postoperative values were close to normal values in most patients. Slight cephalic rotation of the nasal tip was associated with high satisfaction levels, particularly in women.

\section{1) Case 1}

A 42-year-old woman underwent nasal tip plasty with 3D-printed PCL $\left(\right.$ Smart Ball ${ }^{\circledR}$ ) for correction of ptosis of the nasal tip. We observed high levels of patient satisfaction and successful functional outcomes without any complications.

2) Case 2

A 27-year-old woman underwent nasal tip plasty with 3D-printed

Table 2. Plastic surgeons' scores for the outcomes of surgery

\begin{tabular}{cc}
\hline Patient no. & Average \\
\hline 1 & 4.67 \\
2 & 5.00 \\
3 & 3.67 \\
4 & 4.67 \\
5 & 4.33 \\
6 & 4.67 \\
7 & 4.67 \\
8 & 4.33 \\
9 & 4.00 \\
10 & 3.33 \\
11 & 5.00 \\
12 & 4.67 \\
13 & 4.67 \\
14 & 5.00 \\
15 & 4.67 \\
16 & 4.33 \\
17 & 4.33 \\
18 & 4.33 \\
19 & 4.67 \\
20 & 4.33 \\
21 & 4.67 \\
22 & 4.67 \\
Total average (mean \pm SD) & $4.49 \pm 0.41$ \\
\hline SD & \\
\hline standard & \\
\hline
\end{tabular}

$\mathrm{SD}$, standard deviation.

Table 1. Patient satisfaction scores

\begin{tabular}{|c|c|c|c|c|c|c|}
\hline Patient no. & Q1 & Q2 & Q3 & Q4 & Q5 & Q6 \\
\hline Average (mean \pm SD) & $3.59 \pm 0.73$ & $3.50 \pm 0.60$ & $3.82 \pm 0.50$ & $3.73 \pm 0.55$ & $3.55 \pm 0.51$ & $3.82 \pm 0.39$ \\
\hline Total average & \multicolumn{6}{|c|}{3.67} \\
\hline
\end{tabular}

$\mathrm{SD}$, standard deviation. 
Table 3. The preoperative and postoperative measurements of patients

\begin{tabular}{|c|c|c|c|c|c|c|}
\hline \multirow{2}{*}{ Patient no. } & \multicolumn{2}{|c|}{ Nasolabial angle $\left({ }^{\circ}\right)$} & \multicolumn{2}{|c|}{ Columellar-lobular angle $\left({ }^{\circ}\right)$} & \multicolumn{2}{|c|}{ Tip projection (\%) } \\
\hline & PreOP & PostOP & PreOP & Post0P & PreOP & PostOP \\
\hline 1 & 90.50 & 95.60 & 46.70 & 43.00 & 24.70 & 41.00 \\
\hline 2 & 75.00 & 80.00 & 45.50 & 46.00 & 48.00 & 51.50 \\
\hline 3 & 80.00 & 83.00 & 44.00 & 45.50 & 45.20 & 49.00 \\
\hline 4 & 79.00 & 85.50 & 40.00 & 43.00 & 38.00 & 50.20 \\
\hline 5 & 83.00 & 91.00 & 40.00 & 42.00 & 51.00 & 60.20 \\
\hline 6 & 85.60 & 95.00 & 38.50 & 45.00 & 26.00 & 38.50 \\
\hline 7 & 90.00 & 98.50 & 42.80 & 43.00 & 45.00 & 55.00 \\
\hline 8 & 85.00 & 90.50 & 40.50 & 44.00 & 35.00 & 38.00 \\
\hline 9 & 92.00 & 97.30 & 42.00 & 46.50 & 46.00 & 52.50 \\
\hline 10 & 89.80 & 102.00 & 35.00 & 40.20 & 22.00 & 29.00 \\
\hline 11 & 73.50 & 85.00 & 42.00 & 45.80 & 45.00 & 51.30 \\
\hline 12 & 69.00 & 80.50 & 43.00 & 46.00 & 48.00 & 60.10 \\
\hline 13 & 90.00 & 97.10 & 42.00 & 46.60 & 50.00 & 51.20 \\
\hline 14 & 89.00 & 95.50 & 42.00 & 43.00 & 45.80 & 46.10 \\
\hline 15 & 89.00 & 90.50 & 39.00 & 42.50 & 35.70 & 45.00 \\
\hline 16 & 75.00 & 83.00 & 40.00 & 45.00 & 40.50 & 54.00 \\
\hline 17 & 90.00 & 91.50 & 40.00 & 45.00 & 35.00 & 51.80 \\
\hline 18 & 95.80 & 95.00 & 39.00 & 42.00 & 22.90 & 31.00 \\
\hline 19 & 77.00 & 83.50 & 33.00 & 40.00 & 30.00 & 45.80 \\
\hline 20 & 82.50 & 95.00 & 42.00 & 43.50 & 48.20 & 51.00 \\
\hline 21 & 80.00 & 88.80 & 45.90 & 38.45 & 32.10 & 45.22 \\
\hline 22 & 88.00 & 92.80 & 35.65 & 49.31 & 46.10 & 50.00 \\
\hline Total average (mean $\pm S D)$ & $84.03 \pm 7.12$ & $90.75 \pm 6.30$ & $40.84 \pm 3.41$ & $43.88 \pm 2.52$ & $39.10 \pm 9.42$ & $47.61 \pm 8.10$ \\
\hline
\end{tabular}

$\mathrm{OP}$, operation; $\mathrm{SD}$, standard deviation.

PCL (Smart Ball ${ }^{\circledR}$ ) for a deviated nose and tip ptosis. She complained of nasal tip pinching on the 3rd postoperative day, which resolved 1 month postoperatively, and she reported no other complaints. No complications such as infection or warping occurred postoperatively, and the patient was satisfied with the cosmetic outcome (Fig. 8).

\section{Discussion}

Adjusting the nasal tip is the most challenging task during nasal plastic surgery, because the shape of the tip significantly varies among individuals. Therefore, it is difficult to contour the nasal tip to ensure an aesthetically attractive appearance and perform a procedure that suits the needs of each patient. Creation of an aesthetically attractive nasal tip requires accurate measurements to evaluate the existing nasal contour to determine the optimal procedure and materials for the purpose.

Structural changes in the nasal contour and incision and suturing of these tissues commonly require the open approach, involving a columellar incision. This method is associated with longer operation and incision healing times and/or permanent scars, as well as prolonged edema of the nasal tip. Manipulation of a wider area predisposes to structural or functional adverse effects. In contrast, the closed approach is not associated with any visible scar formation. Additionally, because a smaller area is dissected, the operation time is shorter and patients develop lesser edema.

The 3D-printed PCL is a ball-shaped prosthesis that serves as a biological scaffold that allows the growth of tissues within the tiny holes of the scaffold without interfering with blood circulation and therefore enhances the volume of the nasal tip [3,5]. Complete absorption of PCL occurs in approximately 36 months; however, no study has reported long-term follow-up of $>2$ years. Our study is important because our patients underwent longterm follow-up over at least 2 years (mean 36 months) to evaluate the stability of NTP even after PCL absorption.

A limitation of this technique is that it warrants consistent and long-term postoperative follow-up over the course of 4-5 years, because the shape of the PCL-based implant and its orientation with respect to the surrounding cartilage and soft tissues can be altered following absorption of PCL and replacement by autolo- 


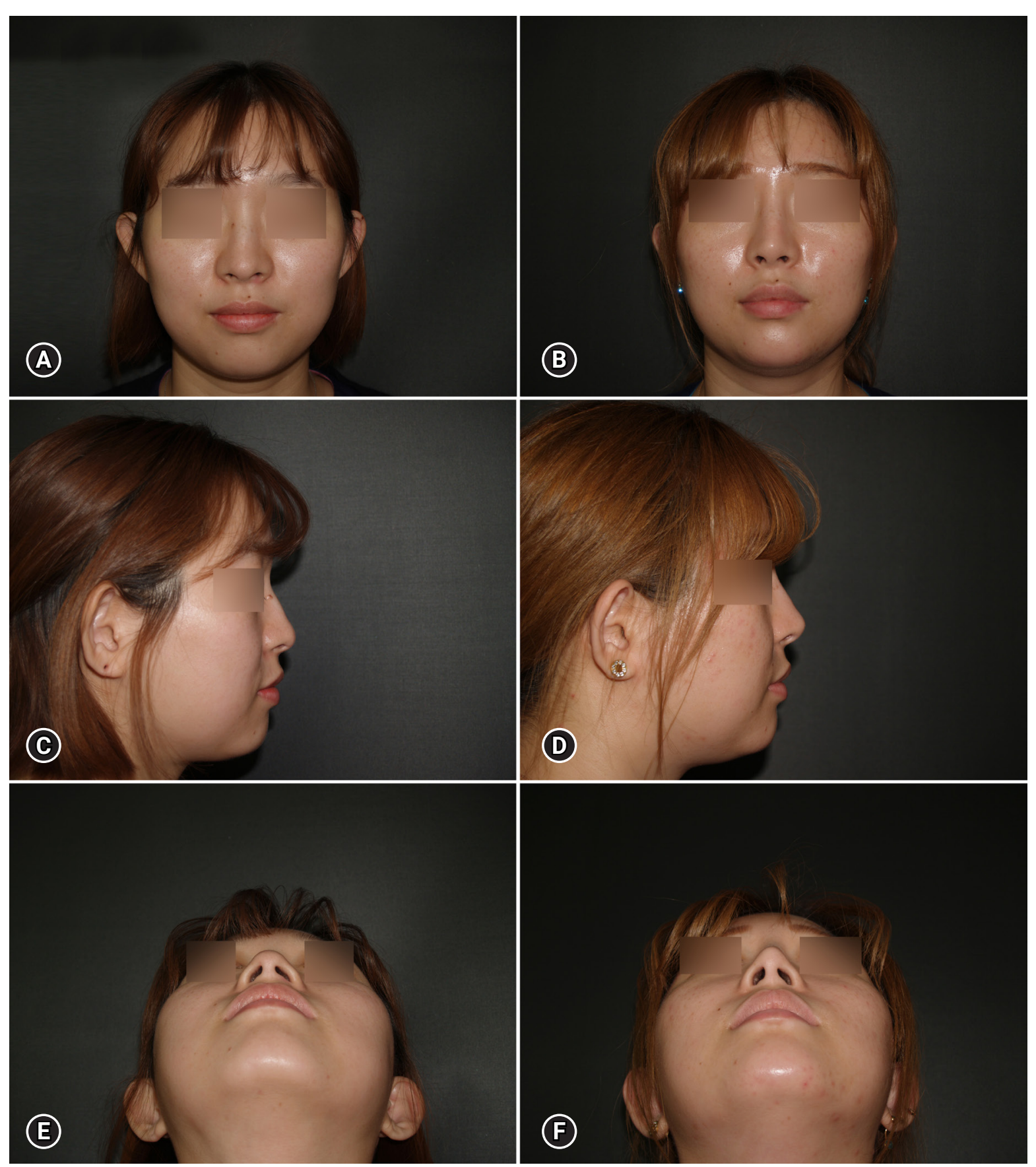

Fig. 8. Representative case of a patient (case 1) show an excellent surgical outcome. Preoperative $(A, C, E)$ and 14-month postoperative (B,

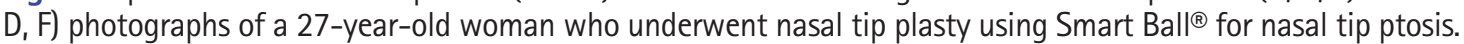

gous tissues [6]. Large-scale studies are warranted in future to confirm the generalizability of this technique. Research in this field is ongoing to obtain more data.

The use of a foreign-body artificial implant for nasal tip contouring increases the risk of complications during long-term follow-up. Following implant fixation, the nasal tip is subjected to high pressure, and this is the point where most complications occur in Asians undergoing rhinoplasty. Complications such as thin- ning of skin and soft tissue, erythema, or inflammation did not occur in any patient in our study.

Previous studies have reported that prostheses made of PCL are structurally complete and can withstand significant dynamic stress [6]. Studies that have reported several-year follow-up in patients receiving PCL prostheses have shown no differences between these patients and those who underwent autologous chondrocyte implantation. Moreover, it has been proved that the me- 
chanical properties of the PCL prostheses correspond to those of cartilage and therefore, this material does not cause skin contraction or permanent scarring, indicating long-term maintenance of the shape of the nasal tip achieved postoperatively [7].

We emphasize that the 3D-printed PCL $\left(\right.$ Smart Ball $\left.{ }^{\circledR}\right)$ technique will significantly benefit patients undergoing nasal tip plasty, because it is a safe procedure that achieves the desired length and volume of the nasal tip without affecting the blood circulation of skin and does not require excessive manipulation.

\section{Acknowledgments}

\section{Conflicts of interest}

No potential conflict of interest relevant to this article was reported.

\section{ORCID}

Joo Hyoung Kim, https://orcid.org/0000-0002-4893-3761

Geon Woo Kim, https://orcid.org/0000-0003-0432-8268

Won Kyung Kang, https://orcid.org/0000-0002-9381-4446

\section{References}

1. Li D, An Y, Yang X. An overview of Asian rhinoplasty. Ann Plast Surg 2016;77(Suppl 1):S22-4.

2. Lam CX, Hutmacher DW, Schantz JT, Woodruff MA, Teoh SH. Evaluation of polycaprolactone scaffold degradation for 6 months in vitro and in vivo.J Biomed Mater Res A 2009;90:906-19.

3. AlHarethy S, Al-Angari SS, Syouri F, Islam T, Jang YJ. Assessment of satisfaction based on age and gender in functional and aesthetic rhinoplasty. Eur Arch Otorhinolaryngol 2017;274:2809-12.

4. Jones AC, Milthorpe B, Averdunk H, Limaye A, Senden TJ, Sakellariou A, et al. Analysis of 3D bone ingrowth into polymer scaffolds via micro-computed tomography imaging. Biomaterials 2004;25:4947-54.

5. Park SH, Yun BG, Won JY, Yun WS, Shim JH, Lim MH, et al. New application of three-dimensional printing biomaterial in nasal reconstruction. Laryngoscope 2017;127:1036-43.

6. Ferril GR, Wudel JM, Winkler AA. Management of complications from alloplastic implants in rhinoplasty. Curr Opin Otolaryngol Head Neck Surg 2013;21:372-8.

7. Welling DB, Maves MD, Schuller DE, Bardach J. Irradiated homologous cartilage grafts. Long-term results. Arch Otolaryngol Head Neck Surg 1988;114:291-5. 